\title{
PERCEPÇÃO DOS GESTORES SOBRE OS MECANISMOS DO SISTEMA DE CONTROLE GERENCIAL: UM ESTUDO COM GESTORES DA BRF FOODSS.A
}

\author{
Cristian Magro ${ }^{1}$ \\ Leossânia Manfroi ${ }^{2}$ \\ Rosane Guze ${ }^{3}$ \\ Carlos Lavarda ${ }^{4}$
}

\section{RESUMO}

Este estudo teve o objetivo identificar a percepção dos gestores, que atuam em uma mesma organização, sobre o uso e a extensão de mecanismos do sistema de controle gerencial. A pesquisa caracteriza-se como um estudo descritivo, de levantamento do tipo survey e com abordagem quantitativa dos dados. A empresa estudada é a BRF FOODS S.A., e a amostra da pesquisa compreendeu 31 gestores de alto escalão, contemplando diretores, supervisores e gerentes. Conclui-se que mesmo a organização tendo uma estrutura administrativa e de produção formalizada, nem todos os mecanismos do sistema de controle gerencial são disseminados e percebidos com uma mesma intensidade pelos gestores de diferentes unidades, como pode ser citado o relatório orçamentário, análise de variações, auditoria externa para qualidade dos padrões e procedimentos, comunicação horizontal, compartilhamento de recursos, sistema de monitoramento das tarefas e autonomia dos gestores.

Palavras-chave: Mecanismos de Gestão; Sistema de Controle; Percepção dos Gestores.

\footnotetext{
1 Doutor em Ciências Contábeis e Administração

${ }^{2}$ Mestre em Ciências Contábeis

3 Uceff - Faculdades

${ }^{4}$ Universidade Federal de Santa Catarina - UFSC
} 


\title{
PERCEPTION OF MANAGERS ON THE MECHANISMS OF MANAGEMENT CONTROL SYSTEM: A STUDY WITH MANAGERS BRF FOODS S.A
}

\begin{abstract}
This study aimed to identify the perception of the managers, who work in the same organization, on the use and extension of mechanisms of the management control system. The research is characterized as a descriptive study, survey type survey and quantitative data approach. The company studied is BRF FOODS S.A., and the research sample comprised 31 senior managers, including directors, supervisors and managers. It is concluded that even the organization having a formalized administrative and production structure, not all the mechanisms of the management control system are disseminated and perceived with the same intensity by the managers of different units, as can be mentioned the budget report, analysis of variations, external audit for quality standards and procedures, horizontal communication, resource sharing, monitoring system of the tasks and autonomy of the managers.
\end{abstract}

Keywords: Management Mechanisms; Control system; Perception Managers.

\section{PERCEPCIÓN DE DIRECTIVOS EN LOS MECANISMOS DE CONTROL DE GESTIÓN: UN STUDIO CON LOS GERENTES DE BRF FOODS S.A.}

\section{RESUMEN}

Este estudio tuvo el objetivo de identificar la percepción de los gestores, que actúan en una misma organización, sobre el uso y la extensión de mecanismos del sistema de control gerencial. La investigación se caracteriza como un estudio descriptivo, de levantamiento del tipo survey y con abordaje cuantitativo de los datos. La empresa estudiada es la BRF FOODS S.A., y la muestra de la encuesta comprendió 31 gestores de alto rango, contemplando directores, supervisores y gerentes. Se concluye que incluso la organización teniendo una estructura administrativa y de producción formalizada, no todos los mecanismos del sistema de control gerencial son diseminados y percibidos con una misma intensidad por los gestores de diferentes unidades, como puede ser citado el informe presupuestario, análisis de variaciones, auditoría externa para calidad de los patrones y procedimientos, comunicación horizontal, compartir recursos, sistema de monitoreo de las tareas y autonomía de los gestores.

Palabras clave: mecanismos de gestión; Sistema de control; La percepción de los directivos. 


\section{INTRODUÇÃO}

É notória a relevância da controladoria para a continuidade das organizações, e suas funções têm evoluído, no intuito de satisfazer a demanda dos usuários da informação. Como pode ser visto, para que as empresas assegurem sua continuidade, é preciso que atendam às necessidades impostas pela sociedade, e portanto, os gestores devem munirse de dados que conduzam ao alcance dos objetivos organizacionais (BORINELLI, 2006).

O desenvolvimento e a evolução dos negócios determinam o surgimento de mecanismos do sistema de controle gerencial. Contudo, a aderência de mecanismos para melhoria da gestão, não tem ocorrido na mesma proporção que sua demanda (HANSEN; OTLEY; VAN DER STEDE, 2003; SOUZA; LISBOA; ROCHA, 2003; GUERREIRO; FREZATTI; CASADO, 2004; FREZATTI, 2005).

Nesse sentido, Frezatti (2005) salienta que os avanços das últimas décadas não solucionaram os gaps dos sistemas de controle gerencial, e portanto, ainda existem questões que necessitam de resolução, inovação e desenvolvimento. A contabilidade gerencial orientada e desenhada apenas para o uso em grandes corporações é um exemplo a ser questionado, visto que os gestores de grandes e/ou médias empresas apontam diferentes percepções e razões para o uso e a extensão dos mecanismos de controle gerencial.

Os argumentos sobre diferenças na percepção dos gestores, advêm do conjunto de atividades desenvolvidas pelas organizações para que assegurem as premissas do planejamento estratégico (ANTHONY; GOVINDARAJAN; DEARDEN, 1998). Nessa perspectiva, os mecanismos do sistema de controle gerencial promovem mudanças no desenvolvimento de curto e longo prazo (Freitas \& Brandão, 2005), e influenciam diretamente nos objetivos organizacionais, no comportamento dos indivíduos e na cultura organizacional (ANTHONY; GOVINDARAJAN; DEARDEN, 1998).

Os sistemas de informações gerenciais são formados por procedimentos e recursos que interagem, de maneira interdependente, com a finalidade de gerar e comunicar informações aos gestores (FREZATTI et al., 2009). Dechow, Granlund e Mouritsen (2006) 
argumentam que os sistemas gerenciais perpassam pela estrutura organizacional e auxiliam nas decisões estratégicas, táticas e operacionais. A utilidade do sistema de controle gerencial pode ser alterada, de acordo com o perfil de cada gestor, e portanto, uma mesma organização pode ter divergência em relação ao uso e a extensão do sistema de controle gerencial.

Diante da inquietação exposta, apresenta-se a questão de pesquisa: qual a percepção dos gestores, que atuam em uma mesma organização, sobre o uso e a extensão de mecanismos do sistema de controle gerencial? 0 objetivo do estudo é identificar a percepção dos gestores, que atuam em uma mesma organização, sobre o uso e a extensão de mecanismos do sistema de controle gerencial.

A pesquisa justifica-se pela escassez de estudos empíricos que expliquem a adoção dos mecanismos de controle gerencial, principalmente em relação a percepção dos gestores que atuam em uma mesma organização (OYADOMARI et al., 2008). A contribuição do estudo é respaldada na compreensão de fatores que influenciam na adoção de instrumentos gerenciais, além de demonstrar como uma grande corporação pode ter diferentes percepções intra-organizacionais sobre os mecanismos postos à sua disposição.

Justifica-se a escolha do setor agroindustrial pelo constante crescimento na demanda nacional e internacional por produtos de origem animal e vegetal, e também pelo destaque do Brasil no pioneirismo de raízes agrícola, terras e clima favoráveis, os quais determinam a alta produtividade no agronegócio brasileiro (MAZZIONI et al., 2007). Diante do crescimento do setor agroindustrial, que determinou a demanda de grande quantidade de informações recebidas e analisadas, diariamente, pelos gestores, se faz necessário estabelecer estratégias de gestão que alcancem todos os níveis organizacionais, sendo que para isso, torna-se preciso compreender a visão dos gestores de diversos níveis sobre os sistemas de gestão utilizados pela organização (RODNISKI; DIEHL; ZWIRTES, 2013).

\section{MECANISMOS DO SISTEMA DE CONTROLE GERENCIAL}


Existem diversos mecanismos úteis ao controle gerencial das organizações, como por exemplo: Activity Based Costing (ABC), Activity Based Management (ABM), Total Quality Management (TQM), Just in Time (JIT), Value Based Management (VBM), Economic Value Added (EVA) e Balanced Scorecard (BSC). Contudo, apesar da existência de sistemas de controle gerencial, altamente, complexos e sofisticados, Guerreiro et al. (2005) apontam que, por vezes, não são suficientes para resolver todos os problemas que envolvem a avaliação do desempenho, remuneração pelo desempenho dos executivos e alocação dos custos. Para auxiliar os sistemas gerenciais na resolução e geração de informações complexas, Atkinson et al. (2018) sugerem a inclusão da análise e controle de informações não-financeiras e subjetivas.

Burns e Scapens (2000) comentam que a contabilidade gerencial estabelece regras e rotinas codificadas, compreendidas, reproduzidas e manifestadas no clima e na cultura organizacional. As informações geradas pelo sistema de controle gerencial influenciam o comportamento dos membros envolvidos no processo de planejamento, coordenação, comunicação, avaliação e decisão (ANTHONY; GOVINDARAJAN, 2001). Nessa perspectiva, o sistema de controle gerencial precisa estar em constante modificação e atualização para que consiga acompanhar as mudanças do mercado, bem como a alteração de comportamento e cultura das pessoas envolvidas no processo organizacional (BROMWICH, 1986).

Freitas e Brandão (2005) comentam que os gestores utilizam de suas aspirações para a identificação e utilização dos mecanismos gerenciais mais adequados a determinadas ocasiões. Nesse sentido, os indivíduos podem influenciar na adaptação dos sistemas de gestão e, por outro lado, os sistemas gerenciais também podem ser capazes de influenciar no comportamento dos indivíduos, sendo que a cultura organizacional pode impactar de maneira positiva e ou negativa em sua congruência (ANTHONY; GOVINDARAJAN, 2001).

A utilização de informações gerenciais no âmbito organizacional não ocorre sistematicamente e depende da percepção dos gestores (SILVA, 2010). 0 uso e a extensão do controle gerencial condiciona-se à constante adaptação (CHENHALL; LANGFIELD- 
SMITH, 1998), e deve ser considerado útil e amplamente aceito, sendo adequado conforme a necessidade dos gestores (GUERREIRO et al., 2005).

Para tanto, a efetividade no direcionamento do sistema de controle gerencial depende da percepção dos gestores sobre os mecanismos postos à sua disposição (AGUIAR; FREZATTI, 2007; AGUIAR; PACE; FREZATTI, 2009). Chenhall (2003) corrobora sobre a necessidade de compreender a visão dos gestores sobre o papel dos mecanismos gerenciais, no intuito de estabelecer corretamente o processo estratégico.

Atkinson et al. (2008, p. 81) mencionam que o sistema gerencial não pode ser esquematizado sem considerar o contexto em que opera, tornando impossível sua padronização, e para alcançar o foco ao qual se propõem, é salutar considerar as diferenças de necessidade informacional (EDMONDS; TSAY; OLDS, 2008).

Para Kober, Ng e Paul (2003), a abrangência do sistema de controle gerencial é relacionada aos mecanismos postos à disposição dos gestores. Tal amplitude é composta por mecanismos de monitoramento dos resultados, controles de custos, controles burocráticos, mecanismos de comunicação e integração, compartilhamento de recursos, controles rígidos, controles profissionais, cultura organizacional e a adaptação (ARCHER; OTLEY, 1991; ABERNETHY; BROWNELL, 1999; KOBER; NG; PAUL, 2003; KOBER; NG; PAUL, 2007).

Simons (2000) comenta que existem mecanismos que oferecem feedback formal para o monitoramento dos resultados. Existem mecanismos que são utilizados pelos gestores para regular as atividades dos subordinados. Além disso, existem controles gerenciais que servem para medir, monitorar os resultados e corrigir os desvios nas medidas de desempenho organizacional.

Kober, Ng e Paul (2003) explicam que o desempenho organizacional relaciona-se com o sistema de metas orçamentárias e de monitoramento dos insumos/matériasprimas. Para Abernethy e Brownell (1999), a alta administração pode utilizar das informações geradas pelo relatório orçamentário para diagnosticar o alcance das previsões, sendo útil para monitorar, coordenar e comunicar as prioridades estratégicas e, em conjunto com os sistemas de recompensa, pode facilitar o controle das prioridades e compromissos estratégicos. 
Os mecanismos do sistema de custos são abordados por Miller e Friesen (1982), pelos quais, salientam que a maioria das organizações utilizam controles de custos mais simplificados. A aparente falta de interesse das organizações para a manutenção de um controle de custos mais abrangente, pode ser explicada pela intensificação de controles gerenciais formais e de relatórios de desempenho, dentre outros (OUCHI, 1979).

Para Abernethy e Brownell (1999), o uso dos mecanismos do sistema de controle gerencial depende criticamente da estabilidade dos processos e atividades. Há exemplo, tem-se o custo padrão, que exige uma visão clara das relações de entrada e saída de insumos e produtos, não podendo ser obtida em atividades com constantes mudanças e com alta incerteza na determinação de tarefas e atividades.

Como pode ser visto, o custeio padrão e o orçamento flexível são moldados para empresas com longos ciclos de produção e padronização nos processos (ABERNETHY; BROWNELL, 1997). As organizações que possuem pouca variedade de tarefas e uma visão clara das relações de produto-insumo podem justificar o desenvolvimento e a utilização de controles tradicionais (PERROW, 1979).

Os mecanismos do sistema de controle burocrático são destinados a vigilância do pessoal e direcionamento dos subordinados às expectativas de superiores. Além disso, contemplam regras, processos, padrões de produção e de qualidade exigidas na condução e eficiência das tarefas (OUCHI, 1979). Uma estrutura rígida de controles e procedimentos pode ser desenvolvida por um conjunto de manuais que alinhem as atividades administrativas e operacionais (ABERNETHY; BROWNELL, 1999).

Para Archer e Otley (1991) o sistema de comunicação e integração é concebido para apoiar as estratégias e assegurar que haja coordenação entre as divisões de negócio.

De acordo com Abernethy e Brownell (1995), a manutenção do negócio está ligada a capacidade da gestão em processar e integrar informações, tornando a organização cada vez mais ágil na resoluções das falhas (ABERNETHY; LILLIS, 1995).

O sistema de compartilhamento dos recursos relaciona-se com a integração dos recursos entre as divisões de uma mesma organização (OUCHI, 1979), fazendo com que não sejam adquiridos, externamente, recursos já existentes. Assim, caso não seja utilizado 
um sistema eficiente de compartilhamento dos recursos, a empresa poderá apresentar ineficiência no aproveitamento dos recursos internos (KOBER; NG; PAUL, 2007).

Já os controles rígidos, em determinadas circunstâncias, podem ser prejudiciais na implementação de diferenciais competitivos. Em organizações que necessitam de constantes adaptações, os controles informais e descentralizados podem oferecer maior eficiência (FIEGENER, 2011). Os controles formais são importantes, mas os controles informais são complementares na medida em que adicionam fatores de cultura organizacional e controle profissional (ARCHER; OTLEY, 1991; ABERNETHY; BROWNELL, 1999).

O controle profissional e a cultura organizacional estão inter-relacionados, na medida em que valores, julgamentos e ética estejam internalizados entre os membros da organização. Desse modo, todo profissional contratado, antes de iniciar as atividades, deverá perpassar pelo processo de aprendizagem organizacional (ABERNETHY; STOELWINDER, 1995; ABERNETHY; BROWNELL, 1999). Abernethy e Brownell (1997) sugerem que as organizações com alta grau de pesquisa e desenvolvimento, necessitam de controles profissionais que sejam eficazes.

Por fim, considera-se que mesmo com a demanda na geração de informações e a criação de mecanismos de controle gerencial (GRANDLUND; LUKKA, 1998), há uma baixa implementação e adaptação (BEUREN; ROEDEL, 2002; HANSEN; OTLEY; VAN DER STEDE, 2003). Além disso, estudos demonstram que há um comportamento mimético na adoção e implementação dos instrumentos de controle gerencial, principalmente em grandes corporações (SIMONS, 2000).

\section{ESTUDOS SOBRE MECANISMOS DO SISTEMA DE CONTROLE GERENCIAL}

Macintosh e Daft (1987) investigaram a relação entre interdependência departamental e a concepção sobre três elementos de um sistema de controle gerencial (funcionamento do orçamento, relatórios estatísticos periódicos, padrões e procedimentos operacionais). Os resultados suportam a hipótese de que a 
interdependência departamental está relacionada com a ênfase colocada em cada elemento do sistema de controle gerencial.

Chenhall e Langfield-Smith (1998) pesquisaram o grau de adoção de controles gerenciais tradicionais por empresas industriais da Austrália. Os achados indicam que a adoção e os benefícios dos controles gerenciais tradicionais foram maiores que as técnicas de controles gerenciais recentemente desenvolvidas. Por fim, grande parte das empresas australianas adotam técnicas gerenciais que enfatizem informações não financeiras de cunho estratégico.

O estudo de Castro et al., (2007) teve como objetivo identificar, na percepção de alunos de pós-graduação, a relevância dos indicadores de gestão. Os achados revelam que os indicadores de fluxo de caixa projetado e de margem líquida foram os mais relevantes, por outro lado, os indicadores citados como mais sofisticados foram: Balanced Scorecard, lucro antes de juros, impostos, depreciação e amortização, e economic value added. Concluíram que a percepção sobre os mecanismos de controles gerenciais varia conforme o perfil do usuário e a região de localização da empresa.

Aguiar e Guerreiro (2008) analisaram os processos de persistência e mudança dos sistemas de controle gerencial, empregando o paradigma institucional em contraposição ao paradigma neoclássico. As reflexões focalizam que os mecanismos do sistema de controle gerencial atuam como instrumento de gestão social, e podem apresentar homogeneidade nos processos de persistência e mudança. Concluem que a substituição de um mecanismos do sistema de controle gerencial pode ser determinada pela sua perda de capacidade em cumprir com o papel de controle social.

Oyadomari et al. (2008) investigaram a intensidade na adoção de artefatos da contabilidade gerencial por empresas brasileiras. Os resultados apontam que o conhecimento mimético, gerado pela socialização, influencia na adoção de novos artefatos da contabilidade gerencial, e as empresas de consultoria exercem papel importante neste processo.

O estudo de Silva (2010) buscou explicar o nível de percepção dos gestores de micro e pequenas empresas sobre a utilidade da informação contábil na tomada de 
decisão. Os resultados revelam que os gestores possuem indiferença sobre a utilidade da informação contábil para o processo decisório.

Fernandes, Klann e Figueredo (2011) analisaram a importância da informação contábil/gerencial para a tomada de decisão, na concepção de gestores. Os resultados indicam que na concepção dos gestores a informação contábil/gerencial é considerada importante para o processo decisório. Além disso, os gestores consideram que no estágio atual, não possuem deficiência de informações para a tomada de decisão.

Frezatti et al., (2012) analisaram o perfil de desenvolvimento de empresas brasileiras sobre os atributos da contabilidade gerencial. Nesse sentido, concluem que os atributos, dependendo do escopo organizacional, influenciam a existência de determinados artefatos da contabilidade gerencial. Caso haja interesse na utilização dos artefatos da contabilidade gerencial, faz-se necessário revisitar toda a estruturação dos atributos.

Rodniski et al. (2013) investigaram o desenvolvimento de um modelo de Tableau de Bord aplicável a área industrial de uma agroindústria de abate e industrialização de suínos e frangos. Os achados demonstram que o modelo pode trazer contribuições para a gestão da agroindústria. Os principais ganhos apontados pelos gestores foram a clareza e simplicidade com que são apresentados os indicadores da empresa.

Beuren, Czesnat e Silva (2014) verificaram o papel da controladoria na adequação das empresas do agronegócio às contingências identificadas nos relatórios anuais. Concluíram que a controladoria exerce papel fundamental no ambiente interno e externo de organizações do agronegócio listadas na Bovespa. Além disso, relatam que a controladoria auxiliou na solução de problemas decorrentes das contingências já identificadas, por intermédio da participação na elaboração de planos de contenção.

\section{PROCEDIMENTOS METODOLÓGICOS}

O elevado grau de verticalização, em função da cadeia produtiva, no setor agroindustrial provoca demandas diferenciadas para os sistemas de controle gerencial (Rodniski et al., 2013). Além disso, as agroindústrias atuam em um mercado competitivo 
com produtos fabricados em série e de forma padronizada, com margens reduzidas. Esses fatores aumentam as exigências de produção e multiplicam a necessidade de unidades produtoras, tornando cada vez mais complexo o processo gerencial (Rodniski et al., 2013).

Diante das evidências, justifica-se a escolha do estudo na BRF FOODS S.A, uma empresa do setor agroindustrial que conta com o apoio de mais de 13 mil produtores integrados, mais de 30 mil fornecedores (4 mil apenas de grãos, farelo e óleos) e 240 mil clientes globais. Nesse sentido, a população da pesquisa compreendeu 100 gestores (entre diretores, supervisores e gerentes) da unidade localizada no Município de Chapecó/SC. A amostra foi delineada considerando o retorno do instrumento de pesquisa, totalizando 31 gestores.

0 instrumento de pesquisa foi baseado em estudos que abordaram os mecanismos do sistema de controle gerencial. 0 quadro 1 demonstra variáveis do instrumento de pesquisa.

Quadro 1 - Instrumento de pesquisa

\begin{tabular}{|c|c|c|}
\hline Grupos & N. & Questões/variáveis \\
\hline \multirow{5}{*}{$\begin{array}{l}\text { Monitoramento } \\
\text { de resultados }\end{array}$} & 01 & $\begin{array}{l}\text { A empresa utiliza relatórios formais mensais de gestão e } \\
\text { desempenho? }\end{array}$ \\
\hline & 02 & $\begin{array}{l}\text { A empresa utiliza relatórios de gestão do consumo de insumos } \\
\text { e materiais diversos? }\end{array}$ \\
\hline & 03 & $\begin{array}{l}\text { A empresa utiliza a avaliação do desempenho para comparar } \\
\text { com o desempenho dos concorrentes? }\end{array}$ \\
\hline & 04 & $\begin{array}{l}\text { A empresa elabora explicações escritas referente os relatórios } \\
\text { orçamentários do período em comparação a períodos } \\
\text { anteriores? }\end{array}$ \\
\hline & 05 & $\begin{array}{l}\text { A tendência entre os resultados reais do período passado e os } \\
\text { resultados do período corrente são monitorados } \\
\text { frequentemente pelos diretores? }\end{array}$ \\
\hline \multirow{3}{*}{$\begin{array}{l}\text { Controles de } \\
\text { custos }\end{array}$} & 06 & A empresa utiliza centros de custo para a tomada de decisões? \\
\hline & 07 & A empresa utiliza a análise da variação orçamentária? \\
\hline & 08 & Você se depara com metas orçamentárias apertadas? \\
\hline \multirow{4}{*}{$\begin{array}{l}\text { Controles } \\
\text { Burocráticos }\end{array}$} & 09 & A empresa utiliza manuais de procedimentos? \\
\hline & 10 & A empresa realiza avaliação formal de pessoal? \\
\hline & 11 & $\begin{array}{l}\text { Existe auditoria interna dos controles internos e relatórios } \\
\text { financeiros? }\end{array}$ \\
\hline & 12 & Existe auditoria dos padrões de procedimentos e qualidade? \\
\hline
\end{tabular}




\begin{tabular}{|c|c|c|}
\hline & 13 & $\begin{array}{l}\text { Existe auditoria independente dos padrões de procedimentos } \\
\text { e qualidade? }\end{array}$ \\
\hline \multirow{4}{*}{$\begin{array}{l}\text { Mecanismos de } \\
\text { Comunicação e } \\
\quad \text { Integração }\end{array}$} & 14 & $\begin{array}{l}\text { Existe comunicação informal (reuniões, contatos pessoais) } \\
\text { das informações de cima para baixo e de baixo para cima na } \\
\text { hierarquia? }\end{array}$ \\
\hline & 15 & $\begin{array}{l}\text { A empresa realiza reuniões interdisciplinares (reuniões com } \\
\text { colaboradores de diferentes áreas)? }\end{array}$ \\
\hline & 16 & $\begin{array}{l}\text { A informação da diretoria para os demais níveis é bem } \\
\text { comunicada? }\end{array}$ \\
\hline & 17 & A informação é bem comunicada em todas as áreas? \\
\hline \multirow{2}{*}{$\begin{array}{l}\text { Compartilhame } \\
\text { nto de recursos }\end{array}$} & 18 & $\begin{array}{l}\text { Existe equipes interdisciplinares (colaboradores de diferentes } \\
\text { áreas para realizar um mesmo projeto)? }\end{array}$ \\
\hline & 19 & $\begin{array}{l}\text { Existe compartilhamento de recursos (diferentes áreas } \\
\text { compartilhando um mesmo equipamento ou colaboradores)? }\end{array}$ \\
\hline \multirow[b]{2}{*}{$\begin{array}{l}\text { Controles } \\
\text { Rígidos }\end{array}$} & 20 & Existe uma forte adesão as regras, políticas ou planos. \\
\hline & 21 & $\begin{array}{l}\text { Sistemas de controle de gestão são usados para monitorar } \\
\text { todas as tarefas em sua área. }\end{array}$ \\
\hline \multirow{2}{*}{$\begin{array}{l}\text { Controles } \\
\text { Profissionais }\end{array}$} & 22 & Você tem um alto grau de autonomia na tomada de decisões? \\
\hline & 23 & $\begin{array}{l}\text { Seus colaboradores possui um alto grau de autonomia no } \\
\text { exercício de suas funções. }\end{array}$ \\
\hline \multirow{2}{*}{$\begin{array}{c}\text { Cultura } \\
\text { Organizacional }\end{array}$} & 24 & $\begin{array}{l}\text { Existe um grande senso de valores, crenças e normas dentro } \\
\text { da empresa. }\end{array}$ \\
\hline & 25 & $\begin{array}{l}\text { Os colaboradores estão comprometidos com os objetivos da } \\
\text { empresa e seus valores. }\end{array}$ \\
\hline \multirow{2}{*}{$\begin{array}{l}\text { Adaptação dos } \\
\text { Controles }\end{array}$} & 26 & $\begin{array}{l}\text { As informações contidas nos relatórios de controle são sempre } \\
\text { confiáveis. }\end{array}$ \\
\hline & 27 & $\begin{array}{l}\text { Os sistemas de controle de gestão são adaptados para atender } \\
\text { as diferentes necessidades das áreas. }\end{array}$ \\
\hline
\end{tabular}

Fonte: Adaptado de Archer e Otley (1991); Abernethy e Brownell (1999); Kober, Ng e Paul (2003); Kober, Ng e Paul (2007).

As questões de número 01, 02, 03, 04, 06, 07, 09, 10, 11, 12, 13, 14, 15, 18 e 19 apresentadas no quadro 1, verificaram a opinião dos gestores sobre a extensão e o uso dos mecanismos do sistema de controle gerencial. A escala likert utilizada nas questões mencionadas foi de cinco pontos variando de "nunca" (1) a "sempre" (5). Por outro lado, as questões de número $05,08,16,17,20,21$, 22, 23, 24, 25, 26 e 27 verificaram a extensão de concordância dos gestores com relação aos aspectos do sistema de controle gerencial. Para tais questões, a escala likert utilizada foi de sete pontos variando de "discordo totalmente" (1) a "concordo totalmente" (7). 
As questões/variáveis (27) utilizadas no instrumento de pesquisa, conforme quadro 1, foram segregadas em 9 grupos, nomeados de: monitoramento de resultados (MR), controles de custos (CC), controles burocráticos (CB), mecanismos de comunicação e integração (CI), compartilhamento de recursos (CREC), controles rígidos (CRIG), controles profissionais (CP), cultura organizacional (CO) e adaptações dos controles (AC).

Os dados coletados foram tabulados em planilhas eletrônicas e analisados por meio da estatística descritiva. Porém, algumas variáveis foram medidas pela escala likert de cinco pontos e outras pela escala de sete pontos. Para efetuar uma análise comparativa de informações entre as questões foi preciso padronizar os dados. Os dados com escala likert de sete pontos foram padronizados para a escala de cinco pontos, utilizando o método de padronização "z", conforme determina Fávero et al. (2009).

Posteriormente, realizou-se a verificação do peso da informação relacionada a extensão e concordância dos mecanismos do sistema de controle gerencial. Para tanto, utilizou-se o cálculo da entropia com o uso de planilhas eletrônicas. A entropia necessita de um ponto de referência, no qual é calculada a entropia da informação, e nesse caso, foi estabelecido o valor 5, tamanho da escala likert normal e da padronizada.

Por fim, foi utilizada a correlação Pearson e o teste de KMO e Bartlett utilizado para confirmar a aplicabilidade da análise fatorial. A análise fatorial foi utilizada para identificar um número relativamente pequeno de fatores comuns que podem ser utilizados para representar relações entre um grande número de variáveis interrelacionadas. A análise fatorial foi aplicada por meio do software SPSS ${ }^{\circledR}$.

\section{ANÁLISE E INTERPRETAÇÃO DOS RESULTADOS}

O objetivo do estudo foi identificar a percepção dos gestores, que atuam em uma mesma organização, sobre o uso e a extensão de mecanismos do sistema de controle gerencial. A Tabela 1 mostra a análise pela estatística descritiva.

Tabela 1 - Análise descritiva da percepção dos gestores sobre os Mecanismos do Sistema de Controle Gerencial

\begin{tabular}{llll} 
Grupos & (N.) & Média & D.P \\
\hline
\end{tabular}




\begin{tabular}{cccc}
\hline & 01 & 4,13 & 0,96 \\
Monitoramento de & 02 & 4,00 & 0,89 \\
Resultados & 03 & 3,84 & 0,86 \\
& 04 & 3,74 & 1,15 \\
Controles de custos & 05 & 4,33 & 0,59 \\
& 06 & 4,81 & 0,60 \\
Controles Burocráticos & 07 & 4,45 & 0,81 \\
& 08 & 4,53 & 0,58 \\
Mecanismos de & 09 & 3,90 & 0,80 \\
Comunicação e Integração & 10 & 3,68 & 0,95 \\
& 11 & 3,77 & 0,92 \\
Compartilhamento de & 12 & 3,94 & 0,73 \\
Recursos & 13 & 3,48 & 1,07 \\
Controles Rígidos & 14 & 4,03 & 0,60 \\
Controles Profissionais & 15 & 3,58 & 0,76 \\
Cultura Organizacional & 16 & 3,80 & 0,80 \\
Adaptação de Controles & 17 & 3,43 & 0,83 \\
& 18 & 3,68 & 0,65 \\
& 19 & 3,06 & 1,18 \\
& 20 & 4,05 & 0,70 \\
Fonte Dados da Pesquisa. & 21 & 3,88 & 0,85 \\
& 22 & 3,56 & 0,77 \\
& 23 & 3,58 & 0,64 \\
& 24 & 3,99 & 0,66 \\
& 25 & 3,88 & 0,63 \\
& 26 & 3,99 & 0,79 \\
& 27 & 3,69 & 0,76 \\
\hline
\end{tabular}

Fonte: Dados da Pesquisa.

No grupo dos mecanismos de monitoramento de resultados (MR), os gestores concordam que a tendência entre os resultados reais do passado e os resultados correntes são monitorados frequentemente pelos gestores, com média de 4,33 (questão 5). Os relatórios formais (relatório de gestão, relatório de desempenho mensal) também são quase sempre utilizados pelos gestores, com média de 4,13 (questão 1). Por fim, os relatórios de consumo dos insumos e materiais diversos também são percebidos como quase sempre utilizados pelos gestores da organização, com uma média de 4,00 (questão 2).

O segundo grupo aborda os mecanismos de controles de custos (CC), e os achados demonstram que os gestores utilizam o centro de custos com média 4,81 (questão 6) e a 
análise de variância com média de 4,45 (questão 7). Além disso, os gestores concordam que são, muitas vezes, deparados com metas orçamentárias apertadas, com média de 4,53 (questão 8).

O terceiro grupo está relacionado aos mecanismos de controles burocráticos (CB), e os gestores percebem como útil a auditoria interna na definição de padrões e procedimentos de qualidade, com média de 3,94 (questão 12). Os manuais de procedimentos também são percebidos como quase sempre utilizados pela organização, com média de 3,90 (questão 9).

Os mecanismos de comunicação e integração (CI) estão no quarto grupo, e os gestores percebem como quase sempre importante a comunicação informal (reuniões, contatos pessoais), no intuito de fluir as informações aos diversos níveis da hierarquia, com média de 4,03 (questão 14). Os gestores concordam que a "comunicação verbal" é utilizada pela diretoria para repassar informações aos diversos níveis da organização, com média de 3,80 (questão 16).

O sexto grupo é relacionado aos controles rígidos (CRIG), e os gestores concordam que existe forte adesão as regras, políticas ou planos previamente estabelecidos, com média de 4,05 (questão 20). Em relação aos mecanismos de cultura organizacional, foi verificado que os gestores concordam sobre a existência de valores, crenças e normas, com média de 3,99 (questão 24).

Com relação aos mecanismos de adaptação dos controles (AC), observa-se que os gestores concordam que as informações contidas nos relatórios de controle são sempre confiáveis, com média de 3,99 (questão 26). Por fim, os mecanismos de compartilhamento de recursos e os mecanismos de controles profissionais não foram percebidos pelos gestores com muita extensão, em relação ao uso e a concordância.

Na sequência, para analisar o peso da informação transmitido pelas questões, foi utilizada a entropia da informação. A Tabela 2 mostra a entropia e o peso em relação as questões/variáveis dos mecanismos do sistema de controle gerencial.

Tabela 2 - Entropia das questões relacionadas as variáveis dos Mecanismos do Sistema de Controle Gerencial

$\begin{array}{llll}\text { Grupos } & \text { (N.) } & \text { Entropia } & \text { Peso } \\ \end{array}$




\begin{tabular}{|c|c|c|c|}
\hline & & $e\left(d_{i}\right)$ & $\overline{\lambda_{i}}$ \\
\hline \multirow{4}{*}{$\begin{array}{c}\text { Monitoramento de Resultados } \\
\text { (MR) }\end{array}$} & 01 & 0,9915 & 0,2030 \\
\hline & 02 & 0,9922 & 0,1869 \\
\hline & 03 & 0,9924 & 0,1845 \\
\hline & 04 & 0,9851 & 0,3591 \\
\hline \multirow{5}{*}{ Controles de Custos (CC) } & 05 & 0,9972 & 0,0667 \\
\hline & 06 & 0,9973 & 0,2556 \\
\hline & 07 & 0,9946 & 0,5129 \\
\hline & 08 & 0,9976 & 0,2314 \\
\hline & 09 & 0,9938 & 0,1340 \\
\hline \multirow{4}{*}{ Controles Burocráticos (CB) } & 10 & 0,9895 & 0,2269 \\
\hline & 11 & 0,9905 & 0,2058 \\
\hline & 12 & 0,9950 & 0,1093 \\
\hline & 13 & 0,9850 & 0,3240 \\
\hline \multirow{4}{*}{$\begin{array}{c}\text { Mecanismos de Comunicação e } \\
\text { Integração (CI) }\end{array}$} & 14 & 0,9968 & 0,1286 \\
\hline & 15 & 0,9934 & 0,2645 \\
\hline & 16 & 0,9935 & 0,2626 \\
\hline & 17 & 0,9914 & 0,3444 \\
\hline \multirow{2}{*}{$\begin{array}{l}\text { Compartilhamento de Recursos } \\
\text { (CREC) }\end{array}$} & 18 & 0,9954 & 0,1674 \\
\hline & 19 & 0,9771 & 0,8326 \\
\hline \multirow{2}{*}{ Controles Rígidos (CRIG) } & 20 & 0,9955 & 0,3871 \\
\hline & 21 & 0,9929 & 0,6129 \\
\hline \multirow{2}{*}{ Controles Profissionais (CP) } & 22 & 0,9931 & 0,6026 \\
\hline & 23 & 0,9954 & 0,3973 \\
\hline \multirow{2}{*}{ Cultura Organizacional (CO) } & 24 & 0,9961 & 0,5143 \\
\hline & 25 & 0,9963 & 0,4857 \\
\hline \multirow{2}{*}{ Adaptação de Controles (AC) } & 26 & 0,9940 & 0,4737 \\
\hline & 27 & 0,9933 & 0,5263 \\
\hline
\end{tabular}

Fonte: Dados da pesquisa.

Os resultados da Tabela 2 demonstram que no grupo de monitoramento de resultados (MR), a questão 5 , monitoramento das tendências entre períodos apresentou a maior entropia com 0,9972 , mostrando onde existe a menor informação, pelo alto grau de concordância que apresentou. Em relação ao peso, a questão 4, explicações escritas em relatórios orçamentários para as mudanças entre os períodos, apresentou peso 0,3591, mostrando que foi a questão do grupo monitoramento de resultados que apresentou maior efeito surpresa nas respostas em relação ao resultado esperado. Nesse caso, os gestores da organização em estudo indicam que as explicações de relatórios 
orçamentários escritos para a ocorrência de mudanças entre períodos não é amplamente aceito, reconhecido ou executado.

Em relação as variáveis do grupo de controles de custos (CC), foi observado que a questão 8, metas orçamentárias apertadas, apresentou a maior entropia com 0,9976, mostrando que os gestores da organização em estudo são frequentemente deparados com metas de orçamento apertadas. Por outro lado, a questão 7 , análise de variância, apresentou maior peso de informação com 0,512. Esse resultado demonstra que os gestores divergem em opinião sobre a realização de análise da variação orçamentária.

Nas variáveis do grupo de controles burocráticos (CB) verifica-se que a questão 12, auditoria interna para qualidade, apresentou maior entropia com 0,9950. Assim, grande parte dos gestores percebem que a auditoria interna é sempre utilizada pela organização para definir padrões de procedimentos de qualidade. A questão 13, auditoria externa para qualidade, apresentou maior peso de informação com $0,3240.0$ resultado demonstra que os gestores divergem sobre a auditoria externa como ferramenta para verificação de padrões e procedimentos de qualidade.

Nos mecanismos de comunicação e integração (CI), a questão 14, importância das comunicações informais, apresentou maior entropia com 0,9968. É possível inferir que os gestores percebem que a organização utiliza a comunicação informal, realizada por meio de reuniões e contatos pessoais, com o objetivo de transmitir informações. Em contrapartida, a questão 17, comunicações horizontais, apresentou maior peso de informação com 0,3444 . Portanto, os gestores possuem divergência sobre a comunicação horizontal, concluindo-se que tal incongruência pode ocasionar falhas de comunicação aos diversos níveis organizacionais.

Em relação ao compartilhamento de recursos (CREC), a questão 18 (grupos de trabalho interdisciplinar) apresentou maior entropia com 0,9954, mostrando que os gestores percebem que a organização realiza grupos de trabalho para interação e elaboração de projetos. Por outro lado, a questão 19, compartilhamento de recursos, apresentou maior peso de informação com 0,8326 , ou seja, há grande divergência de opinião entre os gestores, que pode ser ocasionada por falhas no compartilhamento de recursos entre as diferentes áreas da organização. 
Nas variáveis de controles rígidos (CRIG), verifica-se que a questão 20, adesão a regras, políticas e planos, apresentou maior entropia com 0,9955. Portanto, os gestores possuem maior concordância em relação a existência de uma forte adesão as regras, políticas ou planos estabelecidos. Em contrapartida, a questão 21 apresentou maior disparidade de opinião entre os respondentes, mostrando um maior peso de informação com 0,6129 . Desse modo, os gestores divergem em opinião sobre o uso dos sistemas para o monitoramento das tarefas.

Verifica-se que nos controles profissionais (CP), a questão 23, pessoa de nível mais baixo tem alto nível de autonomia, apresentou maior entropia com 0,9954. Dessa forma, os gestores percebem que os colaboradores, que estão sob suas responsabilidades, possuem alto grau de autonomia em suas funções. No entanto, a questão 22 , os gerentes tem alto grau de autonomia, apresentou maior peso da informação com 0,6026. Os resultados indicam que os gestores oferecem autonomia para os colaborares que estão sob sua responsabilidade, por outro lado, a direção não oferece autonomia para as decisões dos gerentes.

Por fim, verifica-se que os grupos da cultura organizacional (CO) e da adaptação dos controles (AC) possuem pesos de informação próximos, em suas respectivas questões (24 e 25) para CO e (26 e 27) para AC. Conclui-se que em relação aos fatores de senso de valores, crenças e normas, comprometimento dos colaboradores com os objetivos da organização, confiabilidade nas informações contidas nos relatórios de controle e adaptação dos sistemas de controles para atender as necessidades organizações, possuem proximidade na variação de concordância em relação a percepção dos gestores. A Tabela 3 mostra a entropia e o peso da informação entre os grupos dos mecanismos do sistema de controle gerencial.

Tabela 3 - Entropia entre os grupos dos Mecanismos do Sistema de Controle Gerencial

\begin{tabular}{cccc}
\hline N. & Grupos dos Mecanismos do Sistema de Controle de & $\begin{array}{c}\text { Entropi } \\
\text { Gerencial }\end{array}$ & $\begin{array}{c}\text { Peso } \\
\boldsymbol{e}\left(\boldsymbol{d}_{\boldsymbol{i}}\right)\end{array}$ \\
\hline 01 & Monitoramento de Resultados (MR) & 0,9966 & 0,1116 \\
02 & Controles de Custos (CC) & 0,9989 & 0,0347 \\
03 & Controles Burocráticos (CB) & 0,9967 & 0,1088
\end{tabular}




\begin{tabular}{lcll}
04 & Mecanismos de Comunicação e Integração (CI) & 0,9969 & 0,1027 \\
05 & Compartilhamento de recursos (CREC) & 0,9941 & 0,1954 \\
06 & Controles Rígidos (CRIG) & 0,9960 & 0,1305 \\
07 & Controles Profissionais (CP) & 0,9961 & 0,1282 \\
08 & Cultura Organizacional (CO) & 0,9978 & 0,0737 \\
09 & Adaptação de Controles (AC) & 0,9965 & 0,1144 \\
\hline
\end{tabular}

Fonte: Dados da pesquisa.

Verifica-se na Tabela 3 que os grupos de controle de custos (centro de custos, análise de variância e metas orçamentárias apertadas) e cultura organizacional (valores, crenças e normas e comprometido com os objetivos e valores) apresentaram maior entropia com 0,9989 e 0,9978, respectivamente. Desse modo, conclui-se que os controles de custos e a cultura organizacional são os mecanismos do sistema de controle de gestão percebidos com maior concordância pelos gestores da organização. Por outro lado, o compartilhamento de recursos é o grupo de mecanismos do sistema de controle de gestão, que é percebido pelos gestores com maior diversidade de opinião quanto a sua concordância e utilização pela organização.

Além disso, verifica-se que os gestores percebem a extensão, concordância e uso dos grupos de monitoramento de resultados, controles burocráticos, comunicação e integração, controles rígidos, controles profissionais e adaptação dos controles com pouca variação, conforme os pesos de informação obtidos pela entropia.

Para análise fatorial, primeiramente foi elaborada correlação de Pearson entre os grupos dos mecanismos do sistema de controle gerencial, conforme Tabela 4.

Tabela 4 - Matriz de Correlação entre os grupos dos mecanismos do sistema de controle gerencial

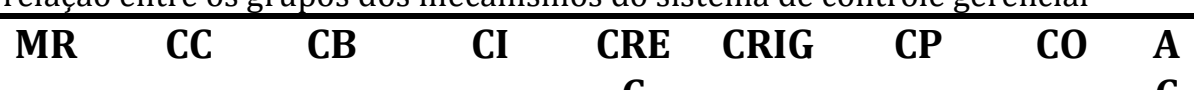

\begin{tabular}{|c|c|c|c|c|}
\hline \multirow[b]{2}{*}{ MR } & Correlaçã & 1 & & \\
\hline & 0 & & & \\
\hline & Sig. & & & \\
\hline \multirow{3}{*}{ CC } & Correlaçã & $0,366^{*}$ & 1 & \\
\hline & o & & & \\
\hline & Sig. & 0,043 & & \\
\hline \multirow{4}{*}{ CB } & Correlaçã & $0,358^{*}$ & 0,24 & 1 \\
\hline & o & & 4 & \\
\hline & Sig. & 0,048 & 0,18 & \\
\hline & & & 7 & \\
\hline
\end{tabular}




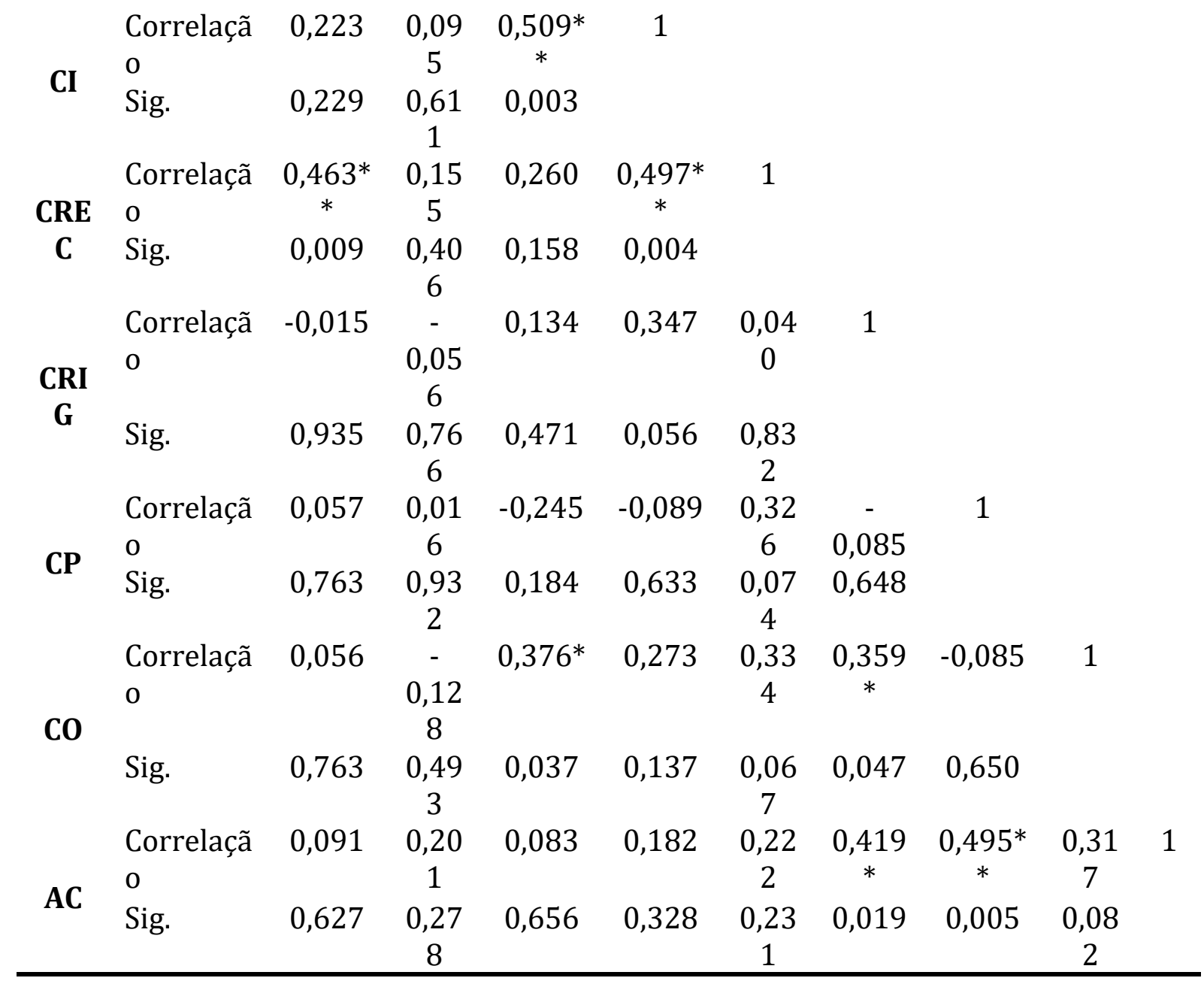

Fonte: Dados da Pesquisa.

Observa-se na Tabela 4 que há alta correlação entre os grupos (MR, CC, CB, CREC), (CB, CI e CO), (CI, CREC), (CRIG, CO, AC), e entre os grupos (CP e AC). Os requisitos iniciais da existência de considerável número de correlação e normalidade multivariada (apenas indícios, já que o teste de normalidade multivariada não foi efetivamente realizado) foram atendidos, o que permite a continuidade da aplicação da análise Fatorial. A Tabela 5 mostra o teste de KMO e Bartlett.

\section{Tabela 5 - Teste de KMO e Bartlett}

Medida Kaiser-Meyer-Olkin de adequação de amostragem.

Teste de esfericidade de Aprox. Qui-quadrado ,672 Bartlett

df. 64,501 
Fonte: Dados da pesquisa.

O teste de KMO varia de 0 e 1, e avalia a adequação da amostra quanto ao grau de correlação parcial entre as variáveis. Assim, o teste de KMO foi de 0,672, apresentando uma qualidade de informação razoável. 0 nível de significância do teste de esfericidade de Bartlett ( $p$-value $=0,000$ ) conduz a evidenciação de correlação entre as variáveis, corroborando com a possibilidade de aplicação da análise fatorial. A Tabela 6 mostra o número de fatores gerados pela análise fatorial.

Tabela 6 - número de fatores gerados pela análise fatorial

\begin{tabular}{|c|c|c|c|c|c|c|c|c|c|}
\hline \multirow[t]{2}{*}{ Compon. } & \multicolumn{3}{|c|}{$\begin{array}{l}\text { Valores Próprios } \\
\text { Iniciais }\end{array}$} & \multicolumn{3}{|c|}{$\begin{array}{c}\text { Somas de Extração de } \\
\text { carregamentos ao } \\
\text { quadrado }\end{array}$} & \multicolumn{3}{|c|}{$\begin{array}{c}\text { Somas rotativas de } \\
\text { carregamentos ao } \\
\text { quadrado }\end{array}$} \\
\hline & Total & $\begin{array}{c}\text { \% } \\
\text { variâ. }\end{array}$ & $\begin{array}{c}\text { \% } \\
\text { cum. }\end{array}$ & Total & $\begin{array}{c}\text { \% } \\
\text { variâ. }\end{array}$ & $\begin{array}{c}\% \\
\text { cum. }\end{array}$ & Total & $\begin{array}{c}\text { \% } \\
\text { variâ. }\end{array}$ & $\begin{array}{c}\% \\
\text { cum. }\end{array}$ \\
\hline 1 & 2,700 & $\begin{array}{c}29,99 \\
7\end{array}$ & $\begin{array}{c}29,99 \\
7\end{array}$ & 2,700 & $\begin{array}{c}29,99 \\
7\end{array}$ & $\begin{array}{c}29,99 \\
7\end{array}$ & 2,153 & $\begin{array}{c}23,92 \\
7\end{array}$ & $\begin{array}{c}23,92 \\
7\end{array}$ \\
\hline 2 & 1,592 & $\begin{array}{c}17,68 \\
5\end{array}$ & $\begin{array}{c}47,68 \\
2\end{array}$ & 1,592 & $\begin{array}{c}17,68 \\
5\end{array}$ & $\begin{array}{c}47,68 \\
2\end{array}$ & 2,041 & $\begin{array}{c}22,67 \\
7\end{array}$ & $\begin{array}{c}46,60 \\
4\end{array}$ \\
\hline 3 & 1,540 & $\begin{array}{c}17,11 \\
6\end{array}$ & $\begin{array}{c}64,79 \\
8\end{array}$ & 1,540 & $\begin{array}{c}17,11 \\
6\end{array}$ & $\begin{array}{c}64,79 \\
8\end{array}$ & 1,638 & $\begin{array}{c}18,19 \\
5\end{array}$ & $\begin{array}{c}64,79 \\
8\end{array}$ \\
\hline 4 & ,991 & $\begin{array}{c}11,00 \\
9\end{array}$ & $\begin{array}{c}75,80 \\
7\end{array}$ & & & & & & \\
\hline 5 & 701 & 7,789 & $\begin{array}{c}83,59 \\
6\end{array}$ & & & & & & \\
\hline 6 & ,592 & 6,573 & $\begin{array}{c}90,16 \\
9\end{array}$ & & & & & & \\
\hline 7 & ,463 & 5,142 & $\begin{array}{c}95,31 \\
1\end{array}$ & & & & & & \\
\hline 8 & ,254 & 2,819 & $\begin{array}{c}98,13 \\
0\end{array}$ & & & & & & \\
\hline 9 & 168 & 1,870 & $\begin{array}{c}100,0 \\
0\end{array}$ & & & & & & \\
\hline
\end{tabular}

Fonte: Dados da pesquisa.

Verifica na Tabela 6 que foram gerados apenas 3 fatores, com percentual de informação do modelo de aproximadamente $64,798 \%$. A Tabela 7 mostra a matriz de componentes rotativa. 
Tabela 7 - Matriz de componente rotativa

\begin{tabular}{cccc}
\hline \multirow{2}{*}{ Grupos } & \multicolumn{3}{c}{ Componente } \\
\cline { 2 - 4 } MR & $\mathbf{1}$ & $\mathbf{2}$ & $\mathbf{3}$ \\
CC & $-0,012$ & 0,806 & 0,029 \\
CB & 0,505 & 0,653 & 0,064 \\
CI & 0,636 & 0,559 & $-0,358$ \\
CREC & 0,303 & 0,437 & $-0,095$ \\
CRIG & 0,750 & 0,640 & 0,332 \\
CP & $-0,167$ & $-0,189$ & 0,139 \\
CO & 0,771 & 0,092 & 0,899 \\
AC & 0,436 & 0,023 & 0,042 \\
\hline
\end{tabular}

Fonte: Dados da pesquisa.

Verifica na Tabela 7 a composição dos três fatores gerados pela análise fatorial. Desse modo, o fator 1 foi composto pelos grupos (CB, CI, CREC, CRIG e CO), o fator 2 pelos grupos (CP e AC) e o fator 3 pelos grupos (MR e CC). Tais fatores podem ser caracterizados de acordo com suas similaridades, e portanto, o fator 1 foi determinado pelo sistema de controle gerencial utilizado para comunicação de políticas, normas e procedimentos que estejam de acordo com os valores para o alcance dos objetivos organizacionais. Já o fator 2 foi determinado pelo sistema de controle gerencial das atividades que envolvem relatórios formais e de controle das atividades e dos recursos. Por fim, o fator 3 foi caracterizado pelo sistema de controle gerencial que oferece subsidio para a autonomia nas decisões e na adaptação dos controles gerenciais.

\section{CONCLUSÕES E RECOMENDAÇÕES}

Diante dos achados, foi possível inferir que os relatórios orçamentários não são amplamente aceitos e reconhecidos pela maioria dos gestores da organização. Desse modo, conforme a concepção de Simons (2000), o monitoramento de resultados da organização poderá ficar prejudicado, na medida em que torna-se incapaz de medir, monitorar e corrigir possíveis desvios. Além disso, o compromisso dos gestores em relação as prioridades estratégicas poderá ser afetado pela falta de monitoramento orçamentário (ABERNETHY; BROWNELL, 1999). 
Os gestores da organização divergem sobre a análise da variação orçamentária. Esse resultado sugere que os processos e tarefas da organização são estáveis diante de mudanças mercadológicas (ABERNETHY; BROWNELL, 1997, 1999). Além disso, foram encontradas divergências da auditoria externa influenciar nos padrões e procedimentos de qualidade.

A organização tende a utilizar guias de procedimentos e manuais para direcionamento das atividades e tarefas (PERROW, 1979), e o fato da auditoria interna ser responsável pela elaboração e disseminação dos procedimentos e manuais, pode ter corroborado para a baixa percepção dos gestores sobre a utilidade da auditoria externa, neste aspecto.

A incongruência sobre a utilização da comunicação horizontal pode gerar falhas de comunicação no alcance de todos os níveis organizacionais, corroborando com Archer e Otley (1991), Abernethy e Brownell (1995) e Abernethy e Lillis (1995), onde mencionam que a ampla utilização da comunicação e integração torna a organização mais ágil, assegurando a coordenação estratégica entre as divisões e todos os níveis de negócio.

Os resultados também indicam que os gestores não percebem que os membros costumam compartilhar (recursos) equipamentos ou colaboradores entre as diferentes áreas da organização e os gestores divergem em opinião sobre o uso dos sistemas de controle gerencial para monitorar todas as tarefas de sua área. Além disso, os gestores oferecem autonomia para os demais colaborares que estão sob sua responsabilidade, e a organização não oferece autonomia para que os próprios gestores tomem decisões.

Diante dos achados, infere-se que os fatores de senso de valores, crenças e normas, comprometimento dos colaboradores com os objetivos da organização, confiabilidade nas informações contidas nos relatórios de controle e adaptação dos sistemas de controles para atender as necessidades organizações, possuem mesma variação de concordância na percepção dos gestores. Percebe-se que a organização tem alto comprometimento com a internalização de valores, crenças e ética dos gestores e colaboradores (ABERNETHY; BROWNELL, 1997).

Além disso, os controles de custos e cultura organizacional foram percebidos com maior concordância pelos gestores, contrapondo a exposição de Miller e Friesen (1982) 
sobre o baixo uso dos controles de custos em detrimento da intensificação dos controles formais. Por outro lado, o compartilhamento de recursos foi percebido pelos gestores com maior diversidade de opinião sobre sua utilização pela organização. De acordo com Kober, Ng e Paul (2007), a organização precisa enfatizar o compartilhamento de recursos entre as divisões, visando diminuir a probabilidade de aquisição de recursos já existentes.

A organização em estudo é de grande porte, com estrutura administrativa e de produção formalizada e bem definida, contudo, apesar disso, nem todos os mecanismos do sistema de controle gerencial são disseminados e percebidos com mesma intensidade pelos gestores que atuam em diferentes unidades (filiais). As conclusões corroboram com as inferências de Frezatti (2005), sobre a existência de divergências na opinião dos gestores, de uma mesma organização, sobre o uso e a extensão dos mecanismos do sistema de controle gerencial. Do mesmo modo, Atkinson et al. (2008) infere sobre a dificuldade de articular controles gerenciais que sejam úteis e aceitos congruente pelos gestores de organizações, complexas, com diversas unidades.

De maneira geral, a organização em estudo precisa considerar a percepção dos gestores na ampliação do uso e na extensão de determinados mecanismos do sistema de controle gerencial (SILVA, 2010). Além disso, o comportamento mimético na utilização de alguns mecanismos do sistema de controle gerencial, pode interferir na percepção de inutilidade por parte dos gestores (SIMONS, 2000).

Por fim, a principal dificuldade da organização, de acordo com Aguiar e Guerreiro (2008), é fazer com que os mecanismos do sistema de controle gerencial cumpram com seu papel, ao passo que existe certa heterogeneidade na percepção dos gestores. Portanto, conclui-se que a organização precisa focalizar no cumprimento dos controles de monitoramento de resultados, controles burocráticos, comunicação e integração, controles rígidos, controles profissionais e adaptações de controles.

Recomenda-se que sejam elaborados estudos futuros com o intuito de verificar se as diferentes percepções podem ter sofrido interferência da localidade e ou região de atuação de cada filial estudada. De acordo com Castro et al. (2007), a região e ou localidade pode determinar diferenças na percepção dos gestores sobre os instrumentos gerenciais. 
Sugere-se observar se a percepção dos gestores sobre os mecanismos do sistema de controle gerencial podem ser determinantes das tipologias estratégicas.

\section{REFERÊNCIAS}

ABERNETHY, M. A.; BROWNELL, P. Management control systems in research and development organizations: the role of accounting, behavior and personnel controls. Accounting, Organizations and Society, v. 22, n. 3-4, p. 233-248, 1997.

ABERNETHY, M. A.; BROWNELL, P. The role of budgets in organizations facing strategic change: an exploratory study. Accounting, organizations and society, v. 24, n. 3, p. 189204, 1999.

ABERNETHY, M. A.; LILLIS, A. M. The impact of manufacturing flexibility on management control system design. Accounting, Organizations and Society, v. 20, n. 4, p. 241-258, 1995.

ABERNETHY, M. A.; STOELWINDER, J. U. The role of professional control in the management of complex organizations. Accounting, Organizations and Society, v. 20, n. 1, p. 1-17, 1995.

AGUIAR, A. B. D.; FREZATTI, F. Escolha da estrutura apropriada de um sistema de controle gerencial: uma proposta de análise. Revista de Educação e Pesquisa em Contabilidade (REPeC), v. 1, n. 3, p. 21-44, 2007

FREZATTI, Fábio et al. Escolha da estrutura apropriada de um sistema de controle gerencial: uma proposta de análise. Revista de Educação e Pesquisa em Contabilidade (REPeC), v. 1, n. 3, p. 21-44, 2008.

AGUIAR, A. B. D.; GUERREIRO, R. Processos de persistência e mudança de sistemas de contabilidade gerencial: uma análise sob o paradigma institucional. Revista Universo Contábil, v. 4, n. 3, p. 06-24, 2008.

AGUIAR, A. B. D.; PACE, E. S. U.; FREZATTI, F. (2009) Análise do Inter-relacionamento das Dimensões da Estrutura de Sistemas de Controle Gerencial: um Estudo Piloto. RACRevista Eletrônica, v. 3 n. 1, p. 1-21, 2009.

ANTHONY, R. N.; GOVINDARAJAN, V.; DEARDEN, J. Management control systems (Vol. 9). Boston, MA: Irwin McGraw-Hill, 1998.

ANTHONY, R. N.; GOVINDARAJAN, V. Management Control Systems, Irwin, Chicago, 2001. 
ARCHER, S.; OTLEY, D. Strategy, structure, planning and control systems and performance evaluation-Rumenco Ltd. Management Accounting Research, v. 2, n. 4, p. 263-303, 1991.

ATKINSON, A. A.; BANKER, R. D.; KAPLAN, R. S.; YOUNG, S. M. Contabilidade Gerencial. 2.ed. São Paulo: Atlas, 2008.

BEUREN, I. M.; ROEDEL, A. 0 uso do custeio baseado em atividades: ABC (Activity Based Costing) nas maiores empresas de Santa Catarina. Revista Contabilidade \& Finanças, v. 13, n. 30, p. 7-18, 2002.

BEUREN, I. M.; CZESNAT, A. F. D. O.; SILVA, M. Papel da controladoria na adequação às contingências ambientais das agroindústrias de carne e derivados listadas na Bovespa. Organizações Rurais \& Agroindustriais, v. 15, n. 3, 2014.

BORINELLI, M. Estrutura Conceitual Básica de Controladoria: sistematização à luz da teoria e da práxis. 2006. 341 f. Tese (Doutorado em Contabilidade) - Departamento de Contabilidade e Atuária da Faculdade de Economia, Administração e Contabilidade, Universidade de São Paulo, São Paulo, 2006.

BURNS, J.; SCAPENS, R. W. Conceptualizing management accounting change: an institutional framework. Management accounting research, v. 11, n. 1, p. 3-25, 2000.

CASTRO, M. C., MURCIA, F. D. R.; BORBA, J. A.; LOESCH, C. Principais indicadores e ferramentas utilizados pelos gestores: uma análise estatística da percepção dos alunos de MBA da Fundação Getúlio Vargas. REGE Revista de Gestão, v. 14, n. 3, p. 49-69, 2007.

CHENHALL, R. H. Management control systems design within its organizational context: findings from contingency-based research and directions for the future. Accounting, organizations and society, v. 28, n. 2, p. 127-168, 2003.

CHENHALL, R. H.; LANGFIELD-SMITH, K. Adoption and benefits of management accounting practices: an Australian study. Management accounting research, v. 9, n. 1, p. 1-19, 1998.

DECHOW, N.; GRANLUND, M.; MOURITSEN, J. Management control of the complex organization: relationships between management accounting and information technology. Handbooks of Management Accounting Research, v. 2, p. 625-640, 2006.

EDMONDS, T. P.; TSAY, B. Y.; OLDS, P. R. Fundamental managerial accounting concepts. McGraw-Hill/Irwin, 2008.

FÁVERO, L. P.; BELFIORE, P.; SILVA, F. L. da; CHAN, B. L. Análise de dados: modelagem multivariada para tomada de decisões. Rio de Janeiro: Elsevier, 5aㅡ - Reimpressão, 2009. 
FERNANDES, F. C.; KLANN, R. C.; FIGUEREDO, M. S. A utilidade da informação contábil para a tomada de decisões: uma pesquisa com gestores alunos. Contabilidade Vista \& Revista, v. 22, n. 3, p. 99-126, 2011.

FIEGENER, Mark K. Matching business-level strategic controls to strategy: Impact on control system effectiveness. Journal of Applied Business Research, v. 10, n. 1, p. 25-34, 2011.

FREITAS, I. A.; BRANDÃO, H. P. Trilhas de Aprendizagem como Estratégia para Desenvolvimento de Competências. Encontro da Associação Nacional dos Programas de Pós-Graduação em Administração- ENANPAD, 29. 2005. Anais... Brasília: ENANPAD, 2005.

FREZATTI, F. Management accounting profile of firms located in Brazil: a field study. Revista de Administração Contemporânea, v. 9, n. SPE2, p. 95-109, 2005.

FREZATTI, F.; ROCHA, W.; NASCIMENTO, A. R. D.; JUNQUEIRA, E. Controle gerencial: uma abordagem da contabilidade gerencial no contexto econômico, comportamental e sociológico. São Paulo: Atlas, 2009.

FREZATTI, F.; JUNQUEIRA, E.; SOUZA, D. D. B.; NASCIMENTO, A. R. D.; RELVAS, T. R. S. Antecedentes da definição do design do sistema de controle gerencial: evidências empíricas nas empresas brasileiras. Brazilian Business Review, v. 9, n. 1, p. 134-155, 2012.

GRANLUND, M.; LUKKA, K. It's a small world of management accounting practices. Journal of management accounting research, v. 10, p. 153, 1998.

GUERREIRO, R.; FREZATTI, F.; CASADO, T. Em busca do entendimento da formação dos hábitos, rotinas e instituições da contabilidade gerencial. In: Congresso USP de Controladoria e Contabilidade, 4., 2004, São Paulo - SP. Anais... São Paulo-SP, 2004.

GUERREIRO, R.; PEREIRA, C. A.; REZENDE, A. J.; AGUIAR, A. B. (2005). Fatores determinantes do processo de institucionalização de uma mudança na programação orçamentária: uma pesquisa ação em uma organização brasileira. Revista de Contabilidade do Mestrado em Ciências Contábeis da UERJ, v. 10, n. 1, 2005.

HANSEN, S. C.; OTLEY, D. T.; VAN DER STEDE, W. A. Practice developments in budgeting: an overview and research perspective. Journal of management accounting research, $\mathrm{v}$. 15, n. 1, p. 95-116, 2003.

KOBER, R.; NG, J.; PAUL, B. Change in strategy and MCS: a match over time?. Advances in Accounting, v. 20, p. 199-232, 2003. 
KOBER, R.; NG, J.; PAUL, B. J. The interrelationship between management control mechanisms and strategy. Management Accounting Research, v. 18, n. 4, p. 425-452, 2007.

MACINTOSH, N. B.; DAFT, R. L. Management control systems and departmental interdependencies: an empirical study. Accounting, Organizations and Society, v. 12, n. 1, p. 49-61, 1987.

MAZZIONI, S.; ZANIN, A.; KRUGER, S. D.; ROCHA, J. L. K. D. A importância dos controles gerenciais para o agribusiness. Revista Catarinense da Ciência Contábil, v. 6, n. 16, p. 09-26, 2007.

MILLER, D.; FRIESEN, P. H. Innovation in conservative and entrepreneurial firms: Two models of strategic momentum. Strategic management journal, v. 3, n. 1, p. 1-25, 1982.

OUCHI, W. G. A conceptual framework for the design of organizational control mechanisms. In: Readings in Accounting for Management Control. Springer US, 1979. p. $63-82$.

OYADOMARI, J. C.; MENDONÇA NETO, O. R. D.; CARDOSO, R. L.; LIMA, M. P. D. Fatores que influenciam a adoção de artefatos de controle gerencial nas empresas brasileiras: um estudo exploratório sob a ótica da teoria institucional. Revista de Contabilidade e Organizações, v. 2, n. 2, p. 55, 2008.

PERROW, C. Organizational analysis: a sociological view. London: Travistock Publications, 1979.

RODNISKI, C. M.; DIEHL, C. A.; ZWIRTES, A. tableau de bord: proposta de aplicação em agroindústria brasileira. Revista Universo Contábil, v. 9, n. 2, p. 63-82, 2013.

SILVA, M. S. D. (2010). Utilidade da informação contábil para tomada de decisões: Um estudo sobre a percepção dos gestores de micro e pequenas empresas da Grande João Pessoa. João Pessoa, 2010. Dissertação (Mestrado em Ciências Contábeis) - Programa Multi-institucional e inter-regional de Pós-graduação em Ciências Contábeis da UnB, UFPB e UFRN, João Pessoa, 2010.

SIMONS, R. Performance measurement and control systems for implementing strategy. Prentice Hall: New Jersey, 2010, 348 p.

SOUZA, M. A. de; LISBOA, L. P.; ROCHA, W. Práticas de contabilidade gerencial adotadas por subsidiárias brasileiras de empresas multinacionais. Revista Contabilidade \& Finanças, v. 14, n. 32, p. 40-57, 2003. 